\title{
Influence and Analysis of Mechanism Magnification of Pneumatic Balance Crane on the Performance of Cylinder
}

\author{
Peipei Feng ${ }^{1, a}$, Qingwei Dong ${ }^{2, b}$ and Xiaoyang Chen ${ }^{3, c}$ \\ ${ }^{1}$ Henan University of Science and Technology, China \\ ${ }^{2}$ Henan University of Science and Technology, China \\ ${ }^{3}$ Henan University of Technology, China \\ a1216843041@qq.com, bhaust_2004@126.com, ccxy125616455@sina.com
}

Keywords: Pneumatic Balance Crane;Cylinder ;Four-Bar linkage;Magnification of mechanism Abstract. Pneumatic balance crane in the process of porter parts can be efficient and precise, cylinder plays a vital role.For a reasonable selection of the required cylinders, by analyzing the motion characteristics of the four-bar linkage and the force analysis of the rod in the pneumatic balance crane, the magnification of the mechanism is introduced.Has obtained the influence of the mechanism magnification on the stroke and the output force of the cylinder ;Solved the problem,finally, given the agency the magnification of the reasonable value.

\section{Introduction}

Pneumatic balance crane is also known as pneumatic balance power manipulator, mainly used for workshop medium-to-large workpiece handling. Compared with the previous electric balance crane and hydraulic transmission balance crane, the pneumatic balance crane can be more fast and stable to move the workpiece from one station to another,to eliminate the phenomenon of tremor and realizes the neutral equilibrium of the workpiece.It saves time, improves efficiency and reduces labor intensity of workers and their security concerns. In order to ensure that the pneumatic balance crane can be smooth and safe in the process of workpiece handling, the selection of the cylinder is particularly important.And the mechanism magnification is closely related to the cylinder,therefore, it is necessary to study and analyze the mechanism magnification of pneumatic balance crane, to get the influence of the cylinder, and find out the method to eliminate the influence of the magnification of mechanism on the performance of the cylinder.

\section{The magnification of the mechanism}

Pneumatic balance crane mainly consists of the fuselage, parallel linkage mechanism, pneumatic drive system and electrical control system. The parallel four-bar linkage mechanism is the main body of pneumatic balance hoist, and it is the key to realize the neutral equilibrium of workpiece. This only analyzes the parallel four-bar linkage of the pneumatic balancing suspension (shown in Fig. 1).

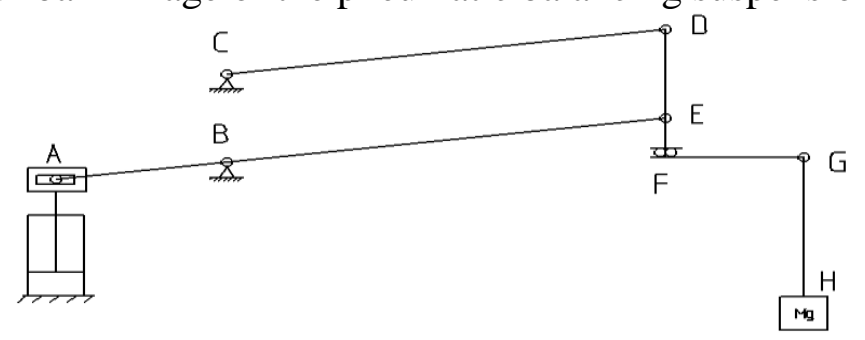

Fig. 1 schematic diagram of parallel four-bar linkage

The motion trajectory of the four-bar linkage is plotted and analyzed, and the two kinds of cases are discussed respectively because of the vertical and horizontal direction of the balance suspension. Analysis of magnification of mechanism in vertical motion of balanced crane

As shown in Fig. 2, the motion diagram of the aerodynamic balance hanging in the vertical limit position (balance suspending of four bar linkage bobbing up and down limit angle is $\varphi$, the $\mathrm{H}_{1}$ is the 
highest point of the workpiece, the $\mathrm{H}_{2}$ is the lowest of the workpiece).
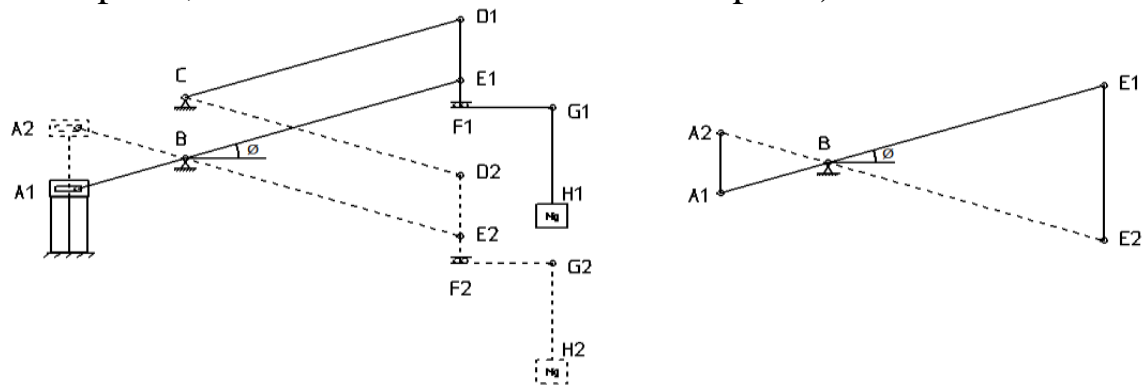

Figure 2 The left is the vertical moving limit position, the right is the limit position analysis sketch

Because the vertical amplitude of the $\mathrm{H}$ point is equal to the vertical amplitude of the $\mathrm{E}$ point, the relationship between the vertical displacement variation of the $\mathrm{E}$ point and the vertical displacement of the A point can be analyzed.

$\because E_{1} B=E_{2} B \quad A_{2} B=A_{1} B \quad \angle E_{1} B E_{2}=\angle A_{1} B A_{2} \therefore \Delta E_{1} B E_{2} \sim \Delta A_{1} B A_{2} \therefore \frac{E_{1} E_{2}}{A_{1} A_{2}}=\frac{E_{1} B}{A_{1} B}=\frac{E_{2} B}{A_{2} B}=\lambda$

The vertical movement of the known E point causes the vertical movement of A point, and the movement of the $E$ point is the $\lambda$ of $A$ point, that is, the vertical movement of the $H$ point is the $\lambda$ times of A point, wherein the $\lambda$ is the magnification of the mechanism, that is, the cylinder stroke is amplified by $\lambda$ on the moving distance of the workpiece. It shows that the magnification of the mechanism plays an important role in the vertical movement of the balance crane and the stroke of the cylinder.

\section{Analysis of magnification of mechanism in horizontal motion of balanced crane}

As shown in Fig. 3, the motion diagram of the aerodynamic balance crane at the horizontal limit position $\left(\mathrm{H}_{1}\right.$ is the most recent point in the horizontal direction of the workpiece is the highest point in the vertical direction, the $\mathrm{H}_{3}$ is the farthest point in the workpiece horizontal direction)
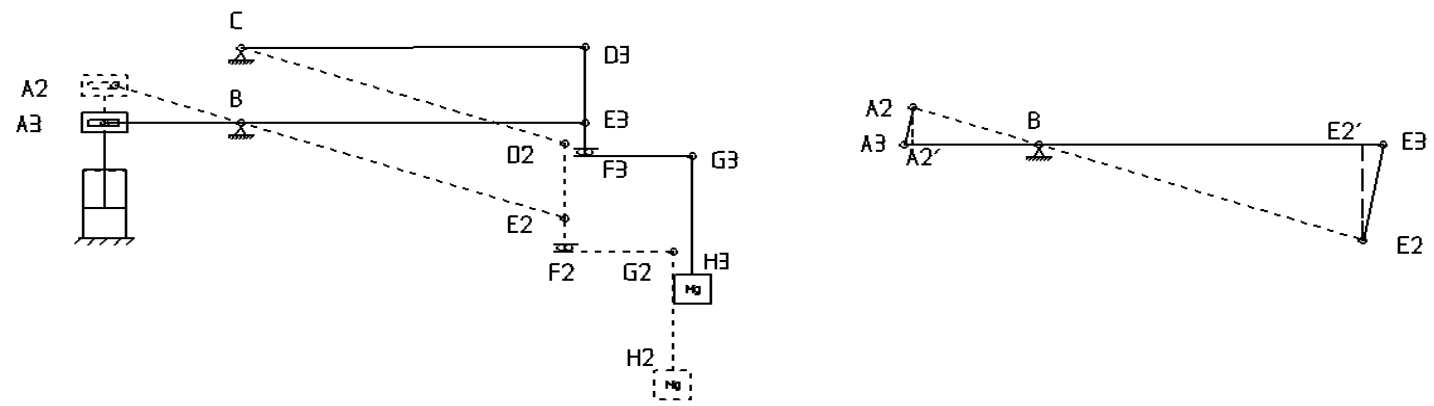

Figure 3 The left is the horizontal moving limit position, the right is the limit position analysis sketch

Similarly, the relationship between the change of horizontal displacement of E point and the change of horizontal displacement of A point is analyzed.

Crosses separately selects $\mathrm{A}_{1}, \mathrm{E}_{1}$ makes $\mathrm{A}_{3} \mathrm{E}_{3}$ perpendicular line $\mathrm{A}_{1} \mathrm{~A}_{1}{ }^{\prime}, \mathrm{E}_{1} \mathrm{E}_{1}{ }^{\prime}$

$\because E_{1} B=E_{3} B \quad A_{1} B=A_{3} B \quad \angle E_{1} B E_{3}=\angle A_{1} B A_{3} \quad \therefore \angle B E_{1} E_{3}=\angle B E_{3} E_{1}=\angle B A_{1} A_{3}=\angle B A_{3} A_{1}$

$\because E_{1} E_{1}^{\prime} \perp A_{3} E_{3} A_{1} A_{1}^{\prime} \perp A_{3} E_{3} \therefore \angle E_{1} E_{1}^{\prime} E_{3}=\angle A_{1} A_{1}^{\prime} A_{3}=90^{\circ}$

$\because \angle B E_{3} E_{1}=\angle B A_{3} A_{1} \therefore \angle E_{1}^{\prime} E_{1} E_{3}=\angle A_{1}^{\prime} A_{1} A_{3} \therefore \Delta E_{1}^{\prime} E_{1} E_{3} \sim \Delta A_{1}^{\prime} A_{1} A_{3}$

$\therefore \frac{E_{1}^{\prime} E_{3}}{A_{1}^{\prime} A_{3}}=\frac{E_{1}^{\prime} E_{1}}{A_{1}^{\prime} A_{1}}=\frac{E_{1} E_{3}}{A_{1} A_{3}} \because \frac{E_{1}^{\prime} E_{1}}{A_{1}^{\prime} A_{1}}=\lambda \therefore \frac{E_{1}^{\prime} E_{3}}{A_{1}^{\prime} A_{3}}=\frac{E_{1}^{\prime} E_{1}}{A_{1}^{\prime} A_{1}}=\frac{E_{1} E_{3}}{A_{1} A_{3}}=\lambda$

$\because R t \Delta E_{1}^{\prime} E_{1} E_{3} \quad \frac{E_{1} E_{1}^{\prime}}{E_{1}^{\prime} E_{3}}=\tan \varphi \therefore \frac{E_{1}^{\prime} E_{1}}{A_{1}^{\prime} A_{1}}=\frac{E_{1}^{\prime} E_{3} \tan \varphi}{A_{1}^{\prime} A_{1}}=\lambda \quad \therefore \frac{E_{1}^{\prime} E_{1}}{A_{1}^{\prime} A_{1}}=\frac{E_{1}^{\prime} E_{3}}{A_{1}^{\prime} A_{1}}=\frac{\lambda}{\tan \varphi}$ 
The horizontal movement of the known E point causes the vertical movement of A point, and the movement of $E$ Point is $\frac{\lambda}{\tan \varphi}$ times of A point, that is, the horizontal movement of $H$ point is $\frac{\lambda}{\tan \varphi}$ times of A point, so the magnification of the mechanism plays an important role in the horizontal movement of the balance hoist and the stroke of the cylinder.

\section{Force analysis of Rod parts}

From Figure 1, the vertical movement of A point is caused by the vertical movement and the horizontal movement of the $\mathrm{H}$ point, if the clamp $\mathrm{H}$ has no load anywhere within the working range, the point $\mathrm{H}$ and point $\mathrm{A}$ are only perpendicular to the opposite force and the resultant joint is zero, and the mechanism can achieve balance.

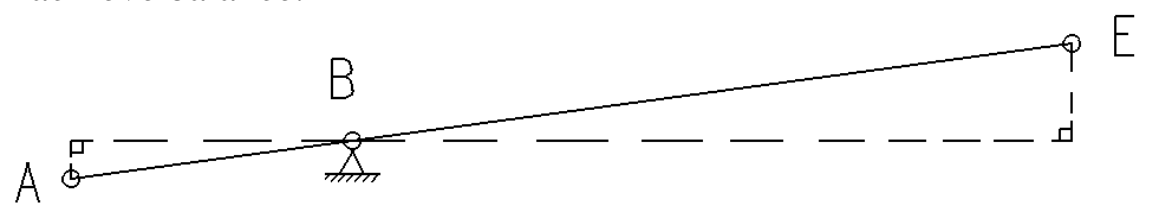

Fig. 4 Analysis diagram of AE rod force of AE rod

$$
\because \sum M(B)=0 \vec{P} \bullet A B \bullet \operatorname{COS} \varphi=\vec{G} \bullet B E \bullet \operatorname{COS} \varphi \frac{B E}{A B}=\lambda \therefore \frac{\vec{P}}{\vec{G}}=\frac{B E \bullet \operatorname{COS} \varphi}{A B \bullet \operatorname{COS} \varphi}=\lambda \therefore \vec{P}=\lambda \vec{G}
$$

Through the analysis of the Rod AE, it is known that regardless of the balance crane load, at what position, A point of the force is the E point $\lambda$ times, that is, A point of the force is the $\mathrm{H}$ point of the $\lambda$ times, the cylinder output force of the load $\lambda$ times.Therefore, the magnification of the mechanism also affects the output force of the cylinder.

\section{The magnification of institutional effects on cylinder}

\section{The influence of magnification on cylinder stroke during moving mechanism}

Through the above analysis shows, the vertical movement of the mechanism is $\lambda$ times the cylinder multiplier, the horizontal movement of the body is $\frac{\lambda}{\tan \varphi}$ times the cylinder stroke, the vertical moving range of the workpiece is determined by the cylinder stroke and the magnification, the horizontal moving range of the workpiece is determined by the angle of the cylinder stroke、 the four-bar linkage rotation and the magnification, So the cylinder's stroke and the vertical and horizontal movement of balance machine have a very close relationship. Only a small force is applied to the workpiece during operation, the workpiece can achieve substantial horizontal movement of the vertical movement; In essence, the stroke of the cylinder is enlarged at point $F$.

The influence of magnification on cylinder output force under any working condition of mechanism

By the rod force analysis shows, $\vec{P}=\lambda \vec{G}$ 。 The output force of the cylinder is related to the magnification of the load and the mechanism, and it is proportional to the magnification of the mechanism. The output force of the cylinder is increases and decreases with the increase of $\lambda$. In essence, the output force of the cylinder is larger than the load $(\lambda-1)$ times.

\section{The influence of institutions magnification on the selection of cylinder}

By the above analysis shows that institutions magnification force affects the stroke of cylinder and output.So agencies magnification is the bigger the better, or as small as possible? A theoretical analysis of the magnification of the institutions is given below:

Institutions magnification affect cylinder stroke and the output power at the same time, so we should analyze the relationship of cylinder stroke and output force firstly. Theoretically, the cylinder stroke have no direct relationship with the output force. But in the actual processing and manufacturing, the longer the cylinder stroke, the greater the difficulty existing in the manufacturing 
process. The cylinder output force decide by the thrust (back) force of the cylinder, The thrust (back) is accomplished by the motion of the piston rod. increase the length of the piston rod, thrust deformation will exist, the longer the deformation is larger, so there is a restriction relationship between the cylinder stroke and the thrust (back), we can also say that there is a restriction relationship between the cylinder stroke and the output force,which confirmed the magnification mechanism of cylinder stroke and output force has great influence.

When $\lambda$ higher, the cylinder stroke is higher, the higher the output force is.

When $\lambda$ lower, the cylinder stroke is lower, the lower the output power is.

But the bigger the $\lambda$, the greater the stroke of the cylinder, the piston rod of the mechanical stress will be too large; the output power is larger, the bore will be larger, and the diameter is too large, not only makes the equipment cumbersome, need high cost, but also increases the consumption of gas, causing a waste of energy. The $\lambda$ is smaller, the stroke of the cylinder is small, can't meet the requirements of practical work; the output force is small, also bore will be smaller, and the diameter is too small, the output power is not enough, cause the cylinder not working properly. Therefore, the magnification of the mechanism can not be too large or too small.

\section{Conclusions}

Based on the above, by analyzing the motion balance of the four-bar linkage of the pneumatic balance crane, the magnification of the mechanism is obtained. Through the further analysis and theoretical derivation of the detailed motion of the four-bar linkage and the force analysis of the bar, the influence of the magnification of the mechanism on the cylinder stroke is obtained: When the mechanism magnification is too large, it will increase the cost and increase the air consumption of the cylinder; the mechanism magnification is too small to cause the cylinder to function normally. Therefore, the choice of institutions magnification is particularly important, neither too large nor too small. By analyzing the application and contact the actual processing, find the ways to deal with the impact of institutional magnification of cylinder: First, to select the value of the mechanism magnification reasonably; Second, to increase or decrease the maximum limit of four-bar linkage swing angle .The above analysis and deduction, selection provides theoretical basis for cylinder, as well as pneumatic balance machine can work efficient and smooth provides a strong guarantee.

\section{References}

[1] Liu Zheng, Analysis and research on influence of mechanism magnification on performance of balance crane,Equipment manufacturing technology, 2010(9),p.50-52.

[2] Jinbin Dai, Meibao Jiang and Zhongrong Ma, Application of constant pressure control in balancing manipulator, Mechanical Design and manufacturing, 2004(2),p.68-72.

[3] Xun Lian and Guangyan Li, Selection design of balancing device for pneumatic balancing manipulator, China academic journal, 2012,p.136-137.

[4] Quanmao He and Fuxian He, Application of parallel four-bar linkage in balance hoist, Coal mine machinery, 2010(10),p.196-197.

[5] Wei Cao, Wei Guo and Wenzhen Yang, The control of tangential and radial operation force of balance hanging, Equipment manufacturing technology, 2011(2),p.144-145.

[6] Hetang $\mathrm{Su}$, Based on MATLAB diesel engine cylinder bore and piston travel optimization design,Journal of Jingdezhen University, 2017(3),p.10-12.

[7] Yu Liu and Guoxin Zhao, No model control device of no friction gas cylinder control simulation, Computer simulation, 2016(3),p.347-350. 